\title{
On a remark of Makowski about perfect numbers
}

\author{
Luis H. Gallardo
}

Luis H. Gallardo received his Ph.D. from the University of Paris 7 in 1978. He is an associate professor at the University of Brest, Brittany, France. He works on arithmetic problems related to polynomials.

\section{Introduction}

A positive number $n>0$ is called perfect if it is equal to the sum $\sigma(n)-n$ of its proper divisors. Some classical papers on perfect numbers were written by Peirce [3], Servais [5], and Sylvester [7] in the 19th century. More recently, Weisstein [9] lists many other properties of the perfect numbers including Makowski's result [2] that 28 is the only even perfect number that exceeds a cube by one. It is natural to wonder whether the result holds for all perfect numbers. More generally, we can ask whether 28 is the only perfect number $n$ that is a sum of two non-negative cubes, say, $n=x^{3}+a^{3}$ with $a \geq 0$ and $x>0$. This seems to be a very difficult question.

The object of this paper is

(a) to prove that 28 is the only even perfect number that is a sum of two positive integral cubes;

(b) to prove (case $a=0$ ) that there are no perfect numbers that are cubes;

(c) to describe a one parameter family of sums $s$ of two cubes such that $s$ is odd and $\sigma(s) \equiv 2(\bmod 4)$

Eine positive natürliche Zahl $n$ heisst bekanntlich vollkommen, falls $n$ gleich der Summe seiner echten positiven Teiler ist; die Zahlen 6 und 28 sind beispielsweise vollkommen. Sätze von Euklid und Euler besagen, dass eine gerade Zahl $n$ genau dann vollkommen ist, wenn $n=2^{p-1}\left(2^{p}-1\right)$ gilt, wobei $2^{p}-1$ und somit auch $p$ Primzahlen sind. Ausgehend von der Gleichung $28=3^{3}+1$ bewies A. Makowski im Jahr 1961, dass die Zahl 28 die einzige gerade vollkommene Zahl ist, die eine Kubikzahl um eins übertrifft. In der vorliegenden Arbeit verallgemeinert der Autor dieses Ergebnis dahingehend, dass er nachweist, dass 28 die einzige gerade vollkommene Zahl ist, die Summe zweier Kubikzahlen ist. 
Now we recall some basic results about perfect numbers. It is well known that any even perfect number $n$ has only two prime factors, so that

$$
n=t(2 t-1)
$$

where $t=2^{p-1}$ for some prime number $p$ such that $2 t-1$ is also prime. Observe that $2 t-1>t$ and that $\operatorname{gcd}(t, 2 t-1)=1$. On the other hand, the most basic result about the form of a possible odd perfect number comes from Euler. Euler [1] proved that odd perfect numbers $n$ have the form

$$
n=y^{4 k+1} z^{2}
$$

with $k \geq 0$ a non-negative integer, $z>0$ a positive integer and $y$ a prime number such that $\operatorname{gcd}(y, z)=1$ and $y \equiv 1(\bmod 4)$. This is indeed an easy consequence of the fact that $\sigma(n) \equiv 2(\bmod 4)$; where $\sigma(n)$ denotes the sum of all positive divisors of $n$.

Let $n$ be an odd perfect number. Touchard [8] proved that either

$$
n \equiv 1 \quad(\bmod 12) \quad \text { or } \quad n \equiv 9 \quad(\bmod 36) .
$$

\section{The only even perfect number that is also a sum of two cubes is 28}

Assume that the even perfect number $n$ is a sum of two cubes:

$$
n=x^{3}+a^{3}=(x+a)\left(x^{2}-a x+a^{2}\right)
$$

for some integers $x>0$ and $a \geq 0$. In particular $a$ and $x$ have the same parity. Let $\delta$ be the discriminant of $x^{2}-(a+1) x+a^{2}-a$. One has $\delta=-\left(3 a^{2}-6 a-1\right)$. Observe that $x^{2}-a x+a^{2}>x+a$ for all $a>2$ since $\delta<0$ if and only if $a>2$. Let us assume now that $a>2$. The case $a \in\{0,1,2\}$ will be considered later. Thus, from (1) we see that $x+a=2^{p-1}$ and $x^{2}-a x+a^{2}=2^{p}-1$ so that

$$
x^{2}-a x+a^{2}=2(x+a)-1 .
$$

Since (4) has integral roots $x$ and $a+2-x$ the discriminant $\Delta=-3 a(a-4)$ of the quadratic

$$
x^{2}-(a+2) x+(a-1)^{2}
$$

must be a perfect square. It is easy to see that $\Delta$ is non-negative exactly when $a \in$ $\{0,1,2,3,4\}$. So it remains to consider the cases $a \in\{3,4\}$. If $a=3$ then $x \in\{4,1\}$. But $n$ is even so $x=1$. Thus, $x=1$ and $a=3$. In other words, we get the perfect number $n=28$. If $a=4$ then $x=3$. This is not possible since $a$ and $x$ have the same parity.

Now we discuss the case $a \in\{1,2\}$. Observe that if $x+a \geq x^{2}-a x+a^{2}$ then we must have $x+a>x^{2}-a x+a^{2}$ since the perfect number $n=(x+a)\left(x^{2}-a x+a^{2}\right)$ is never a square. Take $a=2$. Thus $x+2>x^{2}-2 x+4$. But this is not possible for an integer $x$. It remains only the case $a=1$. In this case we have as before $x+1>x^{2}-2 x+1$. This is true only for $x=1$. So $a=1$ and $x=1$. But $n=x^{3}+a^{3}=2$ is not perfect. Finally, observe that $a=0$ is not possible since 3 does not divide the exponent 1 of the prime $2 t-1$ in $n=t(2 t-1)$. Thus, $n$ is not a cube. This proves the result. 


\section{A perfect number cannot be a cube}

We have just seen that an even perfect number cannot be a cube. We assume in the rest of the section that $n=x^{3}$ is an odd perfect number. We will get a contradiction by considering the equality

$$
\sigma(n)=2 n \quad(\bmod 12)
$$

It follows from (2) that

$$
n=p^{12 k+9} r^{6}
$$

for some non-negative integer $k \geq 0$, for some prime number $p \equiv 1(\bmod 4)$ and for some positive integer $r>0$ such that $\operatorname{gcd}(p, r)=1$. Assume that $r$ can be factored as $r=q_{1}^{\alpha_{1}} \cdots q_{m}^{\alpha_{m}}$ with prime numbers $q_{1}, \ldots, q_{m}$. We have then

$$
2 n=\sigma(n)=\sigma\left(p^{12 k+9}\right) \sigma\left(q_{1}^{6 \alpha_{1}}\right) \cdots \sigma\left(q_{m}^{6 \alpha_{m}}\right) .
$$

\subsection{Case 1: $\operatorname{gcd}(3, n)=1$}

From (3) we get $2 n \equiv 2(\bmod 12)$. Now we compute the value of the right hand side of (5) modulo 12. First of all (3) implies $p \in\{1,5\}(\bmod 12)$ for the Euler prime $p$. So

$$
\sigma\left(p^{12 k+9}\right)=1+p+\ldots+p^{12 k+9} \equiv 12 k+10 \equiv-2 \quad(\bmod 12)
$$

when $p \equiv 1(\bmod 12)$ and

$$
\begin{aligned}
\sigma\left(p^{12 k+9}\right) & =\left(1^{0}+5^{2}+\ldots+5^{12 k+10}\right)+5\left(1^{0}+5^{2}+\ldots+5^{12 k+8}\right) \\
& \equiv(6 k+6)+5(6 k+5)=36 k+31 \equiv 7 \quad(\bmod 12)
\end{aligned}
$$

when $p \equiv 5(\bmod 12)$, since trivially $5^{2} \equiv 1(\bmod 12)$.

Observe that $q_{i}>3$ is an odd prime. So $q_{i} \in\{1,5,7,11\}(\bmod 12)$. Thus, $q_{i}^{2} \equiv 1$ $(\bmod 12)$. In other words $q_{i}^{2 k+1} \equiv q_{i}(\bmod 12)$ and $q_{i}^{2 k} \equiv 1(\bmod 12)$ for any integer $k$. Thus, one has

$$
\sigma\left(q_{i}^{6 \alpha_{i}}\right) \equiv 6 \alpha_{i}+1 \quad(\bmod 12)
$$

when $q_{i} \equiv 1(\bmod 12)$, and

$$
\begin{aligned}
\sigma\left(q_{i}^{6 \alpha_{i}}\right) & =1^{0}+q_{i}^{2}+\ldots+q_{i}^{6 q_{i}}+\left(q_{i}^{1}+q_{i}^{3}+\ldots+q_{i}^{6 q_{i}-1}\right) \\
& \equiv\left(3 \alpha_{i}+1\right)+\left(3 \alpha_{i}\right) q_{i} \equiv 3 \alpha_{i}\left(1+q_{i}\right)+1 \quad(\bmod 12)
\end{aligned}
$$

when $q_{i} \not \equiv 1(\bmod 12)$.

Set $a_{i}=3 \alpha_{i}\left(1+q_{i}\right)+1$. Observe that $a_{i} \equiv 1(\bmod 12)$ when $q_{i} \equiv 7(\bmod 12)$ or when $q_{i} \equiv 11(\bmod 12)$. We have also $a_{i} \equiv 6 \alpha_{i}+1(\bmod 12)$ when $q_{i} \equiv 5(\bmod 12)$.

Moreover, observe that for any integer $x$, either $6 x+1 \equiv 1(\bmod 12)$ or $6 x+1 \equiv 7$ $(\bmod 12)$. Thus, for all $i$ one has

$$
\sigma\left(q_{i}^{6 \alpha_{i}}\right) \in\{1,7\} \quad(\bmod 12) .
$$


Observe that $z \in\{-2,7\}(\bmod 12)$ and $y \in\{1,7\}(\bmod 12)$ implies $z y \in\{-2,1,7\}$ $(\bmod 12)$. Thus,

$$
2 n=\sigma(n)=\sigma\left(p^{12 k+9}\right) \prod_{i=1}^{m} \sigma\left(q_{i}^{6 \alpha_{i}}\right) \in\{-2,1,7\} \quad(\bmod 12) .
$$

This contradicts $(3)$ that gives $2 n \equiv 2(\bmod 12)$; thereby proving the result.

\subsection{Case 2: $\operatorname{gcd}(3, n)=0$}

Observe that

$$
\sigma\left(3^{6 \alpha_{i}}\right)=1+\left(3+3^{2}\right)+3^{2}\left(3+3^{2}\right)+\ldots \equiv 1 \quad(\bmod 12) .
$$

Hence, as before, we get

$$
\sigma(n) \in\{-2,1,7\} \quad(\bmod 12)
$$

But (3) implies that $2 n \equiv 6(\bmod 12)$. So we get the contradiction $\sigma(n) \neq 2 n$. This completes the proof that an odd perfect number cannot be a cube.

\section{Our main result about a family of perfect candidates that are sums of two cubes}

First of all a technical and useful lemma follows:

Lemma 4.1. If for some prime number $p$, for some non-negative integer $k \geq 0$ and for some positive integer $s \geq 1$ one has

$$
p^{4 k+1}=2+3^{2 s-1}
$$

then

$$
k=0 \text {. }
$$

Proof. We choose $r=p, A=2, B=3^{2 s-1}$, and $x=4 k+1$ in order to have

$$
A+B=r^{x} .
$$

We get $P Q=6$ since $P Q$ is the largest squarefree divisor of $A B$. Thus, in order to have the condition $\delta=(A B / P)^{1 / 2}$ is integral fulfilled, we are forced to take $P=6$ and $Q=1$. Hence, $\delta=3^{s-1}$.

It then follows from [4, Theorem 3, p. 219] that

$$
x<\frac{1}{2} Q P^{1 / 2} \ln (P)=\sqrt{6} \frac{\ln (6)}{2}<3 .
$$

But $x$ is odd. So, $x=1$ and $k=0$. Another proof is to choose $x=2 r-1, y=1, n=p$, $z=4 k+1$ in [4, Lemma 2, p. 228] so that we have

$$
3^{x}+2^{y}=n^{z} .
$$

We get as before $k=0$. 
Our main result (whose proof is an immediate corollary of Lemma 4.1 and a check of the formulae) is then:

Theorem 4.2. Let $r>0$ be a positive integer. Define $w(r)=3^{4 r-2}$. Let $k \geq 0$ be a non-negative integer such that the diophantine equation

$$
p(r)^{4 k+1}=2+3^{4 r-1},
$$

has an integral solution $p(r)$. Let also, $a(r)=3 w(r)^{2}-1, m(r)=3 w(r)^{2}+3 w(r)+1$, $n(r)=p(r)^{4 k+1} \cdot 3^{4 r} \cdot m(r)^{2}, x(r)=3^{4 r-1} p(r)^{4 k+1}-a(r)$. Then,

$$
k=0
$$

provided $p(r)$ is a prime number. In all cases we have $w(r) \equiv 1(\bmod 4)$, so that $p(r) \equiv 1(\bmod 4)$, and

a) $p(r)^{4 k+1}=3 w(r)+2$,

b) $x(r)=2 m(r)-1, x(r)=6 w(r)^{2}+6 w(r)+1$,

c) $x(r)+a(r)=3 w(r)(3 w(r)+2)$,

d) $x(r)^{2}-a(r) x(r)+a(r)^{2}=3\left(3 w(r)^{2}+3 w(r)+1\right)^{2}$,

e) $x(r)^{3}+a(r)^{3}=n(r)$.

In other words there is a one parameter family $\{n(r)\}$ of integers such that $n(r) \equiv 1$ $(\bmod 4)$. Moreover if $p(r)$ is prime then $n(r)$ satisfies the necessary condition (2) to be a perfect number. Furthermore, $n(r)$ is a sum of two cubes for each $r$.

It is then of some interest to obtain the $r$ 's for which $p(r)$ is a prime number. We have

$$
n(r) \equiv 1 \quad(\bmod 4) \quad \text { and } \quad \sigma(n(r)) \equiv 2 \quad(\bmod 4) .
$$

A quick computer check gives

a) if $r \equiv 0(\bmod 3)$ then $p(r) \equiv 0(\bmod 7)$;

b) if $r \equiv 2(\bmod 5)$ then $p(r) \equiv 0(\bmod 11)$; etc.

Using a) we constructed a list $L$ of $r$ 's for which $p(r)=2+3^{4 r-1}$ is a prime number. For the moment

$$
L=\{1,4,16,31,35,59,61,79,91,98,283,376,1801,10948,11384,26536\}
$$

contains sixteen elements. We have examined all possible $r$ 's up to 38338 . This took some time, e.g., about 2 hours CPU to test each possible candidate $r$ when $r$ is close to, say, 30000. This was done on a 8 processor linux machine running command line cmaple 11. We do not know if $a_{r}=p(r)$ is a prime number for an infinity of $r$ 's. Compare with sequence $A 134753$ in Sloane's database [6].

Observe that $n(r)$ is perfect if and only if

$$
2 n(r)=2 \cdot p(r) \cdot 3^{4 r} \cdot m(r)^{2}=(p(r)+1) \cdot \sigma\left(3^{4 r}\right) \cdot \sigma\left(m(r)^{2}\right)=\sigma(n(r)) .
$$


We deduce from (9) that if $n(r)$ is perfect then the prime $p(r)$ divides the product $\sigma\left(3^{4 r}\right)$. $\sigma\left(m(r)^{2}\right)$.

Indeed, we checked (in about only 15 seconds) that for all $r \in L$ the corresponding $n(r)$ is not perfect since the prime $p(r)$ does not divide $\sigma\left(m(r)^{2}\right)$.

In order to show that this suffices we claim that if $n(r)$ is perfect then $p(r)$ does not divide $\rho=\sigma\left(3^{4 r}\right)$. To prove the claim assume, to the contrary, that $p(r)$ does divide $\rho$. Then from the definition of $w(r)$ in Theorem 4.2 we obtain that

$$
9 w(r)=3^{4 r} .
$$

But from Theorem 4.2 part a) we get

$$
27 w(r) \equiv-18 \quad(\bmod p(r)) .
$$

So

$$
\rho=\frac{3\left(3^{4 r}\right)-1}{2} \equiv \frac{27 w(r)-1}{2} \equiv \frac{-19}{2} \quad(\bmod p(r)) .
$$

Thus, $p(r)=19$. But 19 , contrary to $p(r)$, is not congruent to $1(\bmod 4)$. This proves the claim.

\section{References}

[1] Euler, L.: De numeris amicabilibus. Reprinted in: Opera posthuma, Euler archive [E798]. http : //www. math. dartmouth. edu/ euler 1 (1862), 85-100.

[2] Makowski, A.: Remark on perfect numbers. Elem. Math. 17 (1962), 109.

[3] Peirce, B.: On perfect numbers. New York Math. Diary 2, XIII (1832), 267-277.

[4] Scott, R.; Styer, R.: On $p^{x}-q^{y}=c$ and related three term exponential Diophantine equations with prime bases. J. Number Theory 105 (2004), 212-234.

[5] Servais, C.: Sur les nombres parfaits. Mathesis 8 (1888), 135.

[6] Sloane, N.J.A.: The On-Line Encyclopedia of Integers Sequences. Published online at: http: //www. research. att. com/ njas/sequences, 2007.

[7] Sylvester, J.J.: Sur les nombres parfaits. Comptes Rendus Paris 106 (1888), 403-405.

[8] Touchard, J.: On prime numbers and perfect numbers. Scripta Math. 19 (1953), 35-39.

[9] Weisstein, E.W.: Perfect Number. http://mathworld.wolfram.com/PerfectNumber.html

Luis H. Gallardo

Mathematics

University of Brest

6, Avenue Le Gorgeu, C.S. 93837

F-29238 Brest Cedex 3, France

e-mail: Luis.Gallardo@univ-brest.fr 